Quim. Nova, Vol. 29, No. 6, 1244-1249, 2006

\title{
ESTUDO TERMOGRAVIMÉTRICO DO EFEITO DA TEMPERATURA E DA ATMOSFERA NA ABSORÇÃO DE DIÓXIDO DE ENXOFRE POR CALCÁRIO
}

\author{
Ivonete Ávila*, Paula M. Crnkovic e Fernando E. Milioli \\ Departamento de Engenharia Mecânica, Escola de Engenharia de São Carlos, Universidade de São Paulo, Av. Trabalhador \\ São-carlense, 400, 13566-590 São Carlos - SP, Brasil \\ Recebido em 1/12/05; aceito em 9/3/06; publicado na web em 11/8/06
}

\begin{abstract}
THERMOGRAVIMETRIC STUDY OF THE EFFECT OF TEMPERATURE AND ATMOSPHERE ON SULFUR DIOXIDE ABSORPTION BY LIMESTONE. Thermogravimetry was applied to investigate the effects of temperature and atmosphere on conversion of sulfur dioxide $\left(\mathrm{SO}_{2}\right)$ absorbed by limestone. Ranges of temperature and particle size were studied, typical of fluidizedbed coal combustion. Isothermal experiments were performed at different temperatures (between 750 and $950{ }^{\circ} \mathrm{C}$ ) under local atmospheric pressure ( $697 \mathrm{mmHg}$ ) in dynamic atmospheres of air and nitrogen. The maximum conversion was $29 \%$ higher in nitrogen atmosphere than in air atmosphere. The optimum conversion temperature was found at $831{ }^{\circ} \mathrm{C}$ in air atmosphere and at $894{ }^{\circ} \mathrm{C}$ in nitrogen atmosphere.
\end{abstract}

Keywords: thermogravimetry; sulfation; limestone.

\section{INTRODUÇÃO}

A queima de combustíveis fósseis com elevados teores de enxofre para produção de energia é reconhecida como a principal fonte de emissão de $\mathrm{SO}_{2}$ para a atmosfera ${ }^{1}$. Entre os combustíveis poluidores de utilização em larga escala, destacam-se os carvões minerais e os óleos pesados derivados do petróleo ${ }^{1}$.

Calcários são largamente empregados para remoção de óxidos de enxofre gerados na combustão de carvões minerais em leito fluidizado. O calcário é injetado na própria câmara de combustão, promovendo a absorção do $\mathrm{SO}_{2}$ in loco, isto é, no mesmo ambiente em que é formado ${ }^{2}$.

O calcário natural, quando submetido a altas temperaturas, absorve energia para calcinar, formando principalmente $\mathrm{CaO}$ com liberação de $\mathrm{CO}_{2}$, aumentando sua porosidade ${ }^{3}$. A reação é mostrada na Equação $1^{4}$

$$
\mathrm{CaCO}_{3}(\mathrm{~s}) \rightarrow \mathrm{CaO}(\mathrm{s})+\mathrm{CO}_{2}(\mathrm{~g}) \quad \Delta \mathrm{H}=+182,1 \mathrm{~kJ} \mathrm{~mol}^{-1}
$$

O aumento da porosidade do calcário após a calcinação é devido à diferença entre o volume molar do absorvente natural $\left(\sim 36,9 \mathrm{~cm}^{3} \mathrm{~mol}^{-1}\right)$ e o volume molar de $\mathrm{CaO}\left(\sim 16,9 \mathrm{~cm}^{3} \mathrm{~mol}^{-1}\right)$ formado ${ }^{3}$. Como o tamanho de partícula do calcário permanece praticamente o mesmo, a porosidade aumenta com a calcinação ${ }^{5,6}$.

Dependendo da temperatura e pressão parcial do $\mathrm{SO}_{2}$, diferentes espécies químicas podem ser produzidas pela reação entre $\mathrm{SO}_{2}$ e $\mathrm{CaO}$. Entre as possíveis rotas de reação, as mais difundidas na literatura são:

Rota 1:

$\mathrm{CaO}(\mathrm{s})+\mathrm{SO}_{2}(\mathrm{~g}) \rightarrow \mathrm{CaSO}_{3}(\mathrm{~s})$

$\mathrm{CaSO}_{3}(\mathrm{~s})+1 / 2 \mathrm{O}_{2}(\mathrm{~g}) \rightarrow \mathrm{CaSO}_{4}(\mathrm{~s})$

Rota 2:

$\mathrm{SO}_{2}(\mathrm{~g})+1 / 2 \mathrm{O}_{2}(\mathrm{~g}) \rightarrow \mathrm{SO}_{3}(\mathrm{~g})$

$\mathrm{CaO}(\mathrm{s})+\mathrm{SO}_{3}(\mathrm{~g}) \rightarrow \mathrm{CaSO}_{4}(\mathrm{~s})$
Rota 3:

$4 \mathrm{CaO}(\mathrm{s})+4 \mathrm{SO}_{2}(\mathrm{~g}) \rightarrow 4 \mathrm{CaSO}_{3}(\mathrm{~s})$

$4 \mathrm{CaSO}_{3}(\mathrm{~s}) \rightarrow 3 \mathrm{CaSO}_{4}(\mathrm{~s})+\mathrm{CaS}(\mathrm{s})$

As reações representadas na rota $1^{7}$ ocorrem a baixas temperaturas, onde o $\mathrm{CaSO}_{3}$ é estável, enquanto as reações da rota $2^{8}$ são dominantes a altas temperaturas $\left(>850{ }^{\circ} \mathrm{C}\right)$, devido à instabilidade do $\mathrm{CaSO}_{3}{ }^{9}$.

Durante a conversão para $\mathrm{CaSO}_{4}$, os poros das partículas de $\mathrm{CaO}$ são preenchidos e saturados com os produtos $\mathrm{CaSO}_{4}, \mathrm{CaSO}_{3} \mathrm{e}$ CaS. Mesmo em comparação com o calcário natural não calcinado, a estrutura física da partícula sulfatada torna-se ainda mais compacta devido à diferença entre os volumes molares de $\mathrm{CaSO}_{4}$ $\left(\sim 46 \mathrm{~cm}^{3} \mathrm{~mol}^{-1}\right)$ e de $\mathrm{CaCO}_{3}\left(\sim 36,9 \mathrm{~cm}^{3} \mathrm{~mol}^{-1}\right)^{3}$.

De modo geral, os parâmetros e as propriedades mais relevantes que afetam o processo de sulfatação de calcários são porosidade, tamanho e distribuição de poros, área superficial interna ${ }^{1,9,10}$, idade geológica, tamanho e densidade das partículas ${ }^{11}$. Um outro aspecto importante a considerar é a presença de componentes químicos nos calcários que possam agir como catalisadores ou envenenadores na sulfatação ${ }^{3}$.

A diminuição da granulometria do calcário proporciona aumento na absorção de $\mathrm{SO}_{2}$, devido à maior área superficial específica. Desta forma, em partículas com granulometrias menores, a sulfatação é mais rápida e supostamente mais completa ${ }^{12}$. Entretanto, o uso de partículas muito pequenas em leito fluidizado é limitado pela elutriação do material, devido a sua baixa velocidade terminal e pelo fato de partículas muito pequenas terem tendência a se aglomerarem ${ }^{13}$. Os estudos termogravimétricos são importantes, como estimativa dos diversos efeitos que possam interferir no processo de absorção do $\mathrm{SO}_{2}$ por calcário, para aplicação posterior em reatores de leito fluidizado.

Um evento que pode ser observado nas curvas TG durante o aquecimento de materiais geológicos, como calcários, é a decrepitação ${ }^{14,15}$. Este é um fenômeno termomecânico provocado por pequenas explosões dentro das partículas, devido à pressão gerada pela evaporação da água no retículo cristalino. Quando ocorre este evento no ensaio TG, as explosões podem fazer com que algumas partículas sejam lançadas para fora do suporte da amostra, indicando nas curvas TG uma perda de massa ${ }^{16}$. 
O efeito da granulometria no fenômeno da decrepitação foi investigado por termogravimetria durante a decomposição térmica de calcários ${ }^{17}$. Foram utilizados calcários calcíticos e dolomíticos com granulometrias médias de 44, 115, 390, 462, 545 e $650 \mu \mathrm{m}$. Os autores não observaram tal fenômeno em calcários dolomíticos. Em calcários calcíticos a decrepitação foi observada somente em granulometrias intermediárias (entre 390 e $462 \mu \mathrm{m}$ ) e entre as temperaturas de 380 e $550{ }^{\circ} \mathrm{C}$.

Os calcários podem ser classificados em função do teor de $\mathrm{MgO}$ presente após a calcinação ${ }^{18}$ : calcítico (0 a $\left.1,1 \%\right)$; magnesiano $(1,1$ a 2,1\%) e, dolomítico (2,1 a 10,8\%). Há controvérsias na literatura quanto à reatividade do $\mathrm{MgO}$ frente ao $\mathrm{SO}_{2}$, pois alguns autores consideram que $\mathrm{o} \mathrm{MgO}$ permanece inerte à sulfatação $\mathrm{o}^{11,19,20}$, enquanto outros sugerem que o $\mathrm{SO}_{2}$ pode ser removido pelo $\mathrm{MgO}$ a temperaturas de $450-500$ até $800^{\circ} \mathrm{C}^{21,22}$.

A reatividade de $\mathrm{MgO}$ frente ao $\mathrm{SO}_{2}$ foi avaliada para este mesmo calcário em estudo de sulfatação ${ }^{16}$. $\mathrm{O}$ autor concluiu que nos testes termogravimétricos, cuja concentração do $\mathrm{SO}_{2}$ foi de $20 \%$, deve-se considerar a fração do $\mathrm{Mg}$ presente na amostra como uma espécie reativa. $\mathrm{O}$ efeito de diferentes tipos de calcário sobre a conversão foi estudado em leito fluidizado de bancada e verificouse que, pelo balanço de massa, a conversão resultou em valores acima de $100 \%$, quando a massa de $\mathrm{Mg}$ foi excluída dos cálculos ${ }^{23}$.

A influência do $\mathrm{O}_{2}$ na absorção do $\mathrm{SO}_{2}$ pelo calcário é assunto de controvérsia na literatura. Alguns resultados indicaram que a maior absorção de enxofre pelo calcário deu-se em atmosfera contendo oxigênio e, a menor, em atmosfera de nitrogênio ${ }^{24}$. Em contrapartida, outros trabalhos indicaram que a conversão aumentou com o decréscimo da fração molar de $\mathrm{O}_{2}$. Porém, observaram que a inclusão de uma pequena quantidade de $\mathrm{O}_{2}$ acelerou a taxa de sulfatação sem que ocorresse uma diminuição significativa na conversão ${ }^{25}$. Foi também observado que as taxas de sulfatação de calcário aparentam ser independentes da concentração de $\mathrm{O}_{2}$ para temperaturas abaixo de $735{ }^{\circ} \mathrm{C}$, e aumentam na presença de $\mathrm{O}_{2}$ acima de $830{ }^{\circ} \mathrm{C}^{3}$.

Neste trabalho emprega-se termogravimetria para se determinar a temperatura ótima de conversão e estudar os efeitos da atmosfera (nitrogênio e ar) no processo de absorção de $\mathrm{SO}_{2}$ por calcário. A faixa de temperatura, entre 750 e $950{ }^{\circ} \mathrm{C}$, e granulometria do calcário $(545 \mu \mathrm{m})$ utilizada são típicas de processos da combustão de carvão em leito fluidizado.

\section{PARTE EXPERIMENTAL}

\section{Condições experimentais}

Utilizou-se um calcário calcítico procedente de Itaú de Minas (MG), cuja composição química parcial (\% massa/massa) é: 35,34 (Ca); 0,35 (Mg); 2,45 (Fe); 0,25 (Al); 0,14 (Sr); 0,09 (K) e 0,52 $(\mathrm{Mn})$. A massa de amostra empregada foi de aproximadamente $10 \mathrm{mg}$ de calcário em cada ensaio. Esta quantidade de massa garante que toda a amostra fique espalhada pelo suporte em uma monocamada, minimizando resistências da transferência de massa nos interstícios entre partículas.

A granulometria média do calcário foi de $545 \mu \mathrm{m}$, obtida por meio de seleção entre duas peneiras sucessivas da série ASTM, de modo a compor a faixa mais estreita possível e minimizar o efeito da distribuição granulométrica.

O suporte de amostra (ou cadinho) utilizado foi de alumina, com 5,8 mm de diâmetro e 2,6 mm de altura. A escolha pelo cadinho de alumina foi feita pelo fato deste material suportar altas temperaturas e não apresentar efeitos catalíticos sobre a reação de sulfatação, quando comparado ao cadinho de platina ${ }^{16,26}$.

As vazões dos gases injetados na balança termogravimétrica (TG) foram de $80 \mathrm{~mL} \mathrm{~min}^{-1}$ para gás de arraste $\left(\mathrm{Ar}\right.$ ou $\left.\mathrm{N}_{2}\right)$ e $20 \mathrm{~mL} \mathrm{~min}^{-1}$ para $\mathrm{SO}_{2}$ (padrão-primário procedente da White Martins). A fração de $\mathrm{SO}_{2}$ no gás reagente ficou em $20 \%$ da atmosfera total.

Os experimentos foram realizados em uma balança TG (Shimadzu - modelo TGA-51). A amostra (calcário natural) foi colocada no cadinho e este introduzido no forno da balança TG. Inicialmente foi feita a calcinação da amostra em atmosfera dinâmica de $\mathrm{Ar}$ ou $\mathrm{N}_{2}$, com razão de aquecimento de $30^{\circ} \mathrm{C} \mathrm{min}{ }^{-1}$ até a temperatura desejada. Após a calcinação e 5 min de estabilização, o gás $\mathrm{SO}_{2}$ foi injetado no forno da balança sob condição isotérmica durante $20 \mathrm{~min}$.

Foram consideradas cinco temperaturas diferentes nos ensaios isotérmicos de sulfatação $\left(750,800,850,900\right.$ e $\left.950{ }^{\circ} \mathrm{C}\right)$, e para cada ensaio foram feitas quatro repetições. Nos ensaios isotérmicos em temperaturas abaixo de $850{ }^{\circ} \mathrm{C}$, foi necessário levar a temperatura até $850{ }^{\circ} \mathrm{C}$ para promover a calcinação completa da amostra. Nestes experimentos, a razão de aquecimento foi de $30{ }^{\circ} \mathrm{C} \min ^{-1}$ até $850{ }^{\circ} \mathrm{C}$, permanecendo nesta temperatura por $5 \mathrm{~min}$, quando então iniciou-se o resfriamento com razão de $30^{\circ} \mathrm{C} \mathrm{min}^{-1}$ até a temperatura desejada. Em seguida, procedeu-se conforme descrito anteriormente.

A Figura 1 mostra o esquema do módulo termogravimétrico, sistema de aquisição de dados e trajeto dos gases até o forno da balança TG. Através do sistema da balança flui apenas o gás de arraste $\left(\mathrm{N}_{2}\right.$ ou Ar), controlado por um rotâmetro interno da balança TG. Quanto ao $\mathrm{SO}_{2}$, este não entra em contato com o mecanismo da balança, sendo injetado diretamente no forno, com vazão controlada por um regulador de fluxo Aalborg específico para $\mathrm{SO}_{2}$.

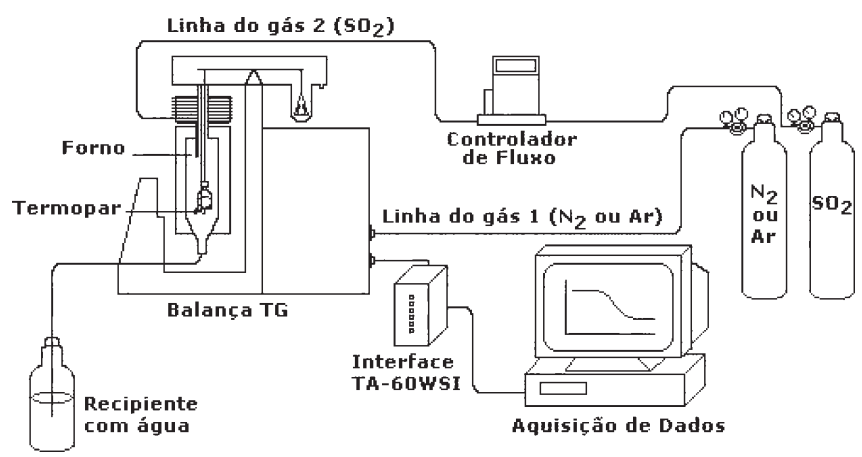

Figura 1. Sistema termogravimétrico: linhas dos gases, aquisição de dados e descarte do rejeito

São necessários cuidados especiais no uso de $\mathrm{SO}_{2}$ nos ensaios termogravimétricos, de forma a evitar danos tanto ao analista quanto ao equipamento. É necessário o uso de óculos de proteção e máscara para gases ácidos, por se tratar de um gás tóxico, e a exaustão do ambiente deve permanecer ligada durante todo o experimento. Devese desligar a válvula de saída do cilindro de $\mathrm{SO}_{2}$ imediatamente após o término de cada ensaio. $\mathrm{O}$ gás de arraste deve permanecer ligado até o completo resfriamento do equipamento, para que ocorra uma limpeza completa do sistema. $\mathrm{O}$ excesso de gás que não reage é eliminado do sistema na parte inferior da balança TG, onde é conectada uma mangueira de silicone com sua extremidade imersa em um recipiente contendo água (rejeito). Periodicamente (de 10 a 15 ensaios) este rejeito é neutralizado e, então, descartado.

\section{Cálculo da conversão}

Pode-se definir a conversão de um reagente $i$ como a relação quantitativa entre o número de moles da espécie alimentada no processo e o número de moles desta mesma espécie que efetivamente reage. A conversão pode ser apresentada na forma percentual: 
Conversão $(\%)=\frac{\text { moles de } i \text { que reagem }}{\text { moles de } i \text { alimentados no processo }} \times 100$

Em um processo operando em regime estacionário, a quantidade de moles da espécie química $i$ que reage é igual à diferença entre a quantidade de moles de $i$ que entram no processo $\left(n_{e}\right)$ e a quantidade de moles de $i$ que saem $\left(n_{s}\right)$. Desta forma, em regime estacionário a conversão $(X)$ é dada pela Equação 9

$x=\frac{n_{e}-n_{s}}{n_{e}} \times 100$

Cada mol de $\mathrm{CaO}$ e $\mathrm{MgO}$ do calcário calcinado contém 1 mol de $\mathrm{Ca}$ e $\mathrm{Mg}$ respectivamente, e cada mol de $\mathrm{CaO}$ e $\mathrm{MgO}$ absorve $1 \mathrm{~mol}$ de $\mathrm{SO}_{2}$. Assim:

$X=\frac{n_{\mathrm{SO}_{2}}}{n_{\mathrm{Ca}}+n_{\mathrm{Mg}}} \times 100$

sendo: $n_{\mathrm{SO} 2}$ a quantidade molar de $\mathrm{SO}_{2}$ absorvida; $n_{\mathrm{Ca}}$ a quantidade molar de $\mathrm{Ca}$ e $n_{M g}$ a quantidade molar de $\mathrm{Mg}$ na amostra.

Nota-se na composição química parcial do calcário calcítico de Itaú de Minas que a fração de magnésio da amostra é muito menor que a fração de cálcio. Entretanto, nos cálculos de conversão no processo de absorção de $\mathrm{SO}_{2}$ por calcário, consideram-se como espécies reativas o $\mathrm{Ca}$ e o $\mathrm{Mg}$ presentes no calcário.

A injeção de $\mathrm{SO}_{2}$ na balança termogravimétrica promove a formação de $\mathrm{CaSO}_{4}$ preferencialmente, ocorrendo o aumento de massa. Assim, a variação de massa do calcário calcinado devido à sulfatação $\left(\Delta M_{S}\right)$ é dada por:

$\Delta M_{\mathrm{S}}=M_{\mathrm{SO}_{2}}+M_{\mathrm{O}_{2}}=n_{\mathrm{SO}_{2}} W_{\mathrm{SO}_{2}}+n_{\mathrm{O}_{2}} W_{\mathrm{O}_{2}}$

sendo $W_{\mathrm{SO}_{2}}$ e $W_{\mathrm{O}_{2}}$ as massas molares do $\mathrm{SO}_{2}$ e do $\mathrm{O}_{2}$, respectivamente.

Dadas as reações:

$\mathrm{CaO}+\mathrm{SO}_{2}+1 / 2 \mathrm{O}_{2} \rightarrow \mathrm{CaSO}_{4}$

$\mathrm{MgO}+\mathrm{SO}_{2}+1 / 2 \mathrm{O}_{2} \rightarrow \mathrm{MgSO}_{4}$

tem-se que:

$\frac{n_{\mathrm{SO}_{2}}}{n_{\mathrm{O}_{2}}}=\frac{1}{1 / 2} \log : n_{\mathrm{O}_{2}}=1 / 2 n_{\mathrm{SO}_{2}}$

Substituindo-se a Equação 14 na Equação 11, tem-se:

$\left.\Delta M_{S}=n_{S_{2}}\left(W_{S_{2}}+1 / 2 W_{\mathrm{O}_{2}}\right) \quad \operatorname{logo}: n_{S_{2}}=\frac{\Delta M_{S}}{\left(W_{S_{2}}+1 / 2 W_{\mathrm{O}_{2}}\right.}\right)$

Os números de moles de cálcio $\left(n_{C a}\right)$ e magnésio $\left(n_{M g}\right)$ disponíveis na amostra são dados por:

$n_{C a}+n_{M g}=M_{A}\left(\frac{Y_{C a}}{W_{C a}}+\frac{Y_{M g}}{W_{M g}}\right)$

sendo $M_{A}$ a massa inicial da amostra em mg, $Y_{C a}$ e $Y_{M g}$ as frações mássicas de $\mathrm{Ca}$ e $\mathrm{Mg}$ presentes na amostra e $W_{C a}$ e $W_{M g}$ as massas atômicas de $\mathrm{Ca}$ e $\mathrm{Mg}$, respectivamente.

Substituindo-se as Equações 15 e 16 na Equação 10, obtém-se a Equação para cálculo da conversão em atmosfera de ar:
$X=\frac{\frac{\Delta M_{S}}{W_{\mathrm{so}_{2}}+1 / 2 W_{\mathrm{O}_{2}}}}{M_{A}\left(\frac{Y_{\mathrm{Ca}}}{W_{\mathrm{Ca}}}+\frac{Y_{M g}}{W_{M g}}\right)} \times 100$

Em atmosfera de nitrogênio tem-se:

$X=\frac{\frac{\Delta M_{S}}{W_{\mathrm{so}_{2}}}}{M_{A}\left(\frac{Y_{C a}}{W_{C a}}+\frac{Y_{M g}}{W_{M g}}\right)} \times 100$

A variação da massa do calcário calcinado durante a sulfatação $\left(\Delta M_{S}\right)$ é obtida nos ensaios termogravimétricos como:

$\Delta M_{S}=M_{F}-M_{C}$

sendo $M_{F}$ a massa final após a sulfatação completa em relação à massa do calcário calcinado $\left(M_{C}\right)$. Os valores de $M_{A}, M_{F}$ e $M_{C}$ são dados obtidos nas curvas TG.

É conveniente comparar o espalhamento dos resultados em diferentes condições de valores médios. Para tal, normaliza-se o desvio padrão em função da média aritmética das medidas, definindose um coeficiente de variação $(C V)^{27}$, dado por:

$C V=\frac{\sigma}{\bar{X}} \times 100$

sendo $\bar{X}$ a média aritmética e $\sigma$ o desvio padrão, dados por:

$\bar{x}=\frac{\sum_{i=1}^{n} x_{i}}{N}$

$\sigma=\sqrt{\frac{\sum_{i=1}^{n}\left(X_{i}-\bar{X}\right)^{2}}{N-1}}$

sendo $X_{i}$ os valores obtidos em cada repetição dos ensaios e $N$ a quantidade de repetições.

Quanto menor o $C V$, mais preciso é o resultado obtido no experimento. Os valores de $C V$ são considerados baixos quando inferiores a $10 \%$, médios quando de 10 a $20 \%$, altos quando de 20 a $30 \%$, e muito altos quando superiores a $30 \%{ }^{28}$.

\section{RESULTADOS E DISCUSSÃO}

A Figura 2 mostra curvas obtidas na calcinação seguida de sulfatação para um dos ensaios termogravimétricos realizados. São mostrados os perfis TG, DTG e razão de aquecimento $\left(30{ }^{\circ} \mathrm{C} \mathrm{min}^{-1}\right)$. Os indicadores [1] e [2] sobre a curva TG sinalizam os patamares entre os quais se desenvolve a perda de massa devido à calcinação. Após a calcinação completa, o $\mathrm{SO}_{2}$ é injetado na atmosfera do forno, e a sulfatação se desenvolve. $\mathrm{O}$ ganho de massa na sulfatação ocorre entre os patamares [2] e [3].

Foram feitos quatro ensaios termogravimétricos para cada temperatura escolhida $\left(750,800,850,900\right.$ e $\left.950{ }^{\circ} \mathrm{C}\right)$, em cada atmosfera estudada (ar sintético e nitrogênio). A Figura 3 apresenta um conjunto destes testes para a temperatura de $900{ }^{\circ} \mathrm{C}$ em atmosfera de nitrogênio. Nota-se que em todas as curvas TG apresentadas na Figura 3 há uma perda de massa entre 20 e $30 \mathrm{~min}$, correspondente à liberação de $\mathrm{CO}_{2}$.

Na Figura 4 estão representadas as curvas da derivada temporal, ou curvas DTG, referentes às curvas TG apresentadas na Figura 3. Foram obtidas as curvas TG e DTG para todas as temperaturas nas duas atmosferas estudadas, como já descrito ${ }^{29}$. 


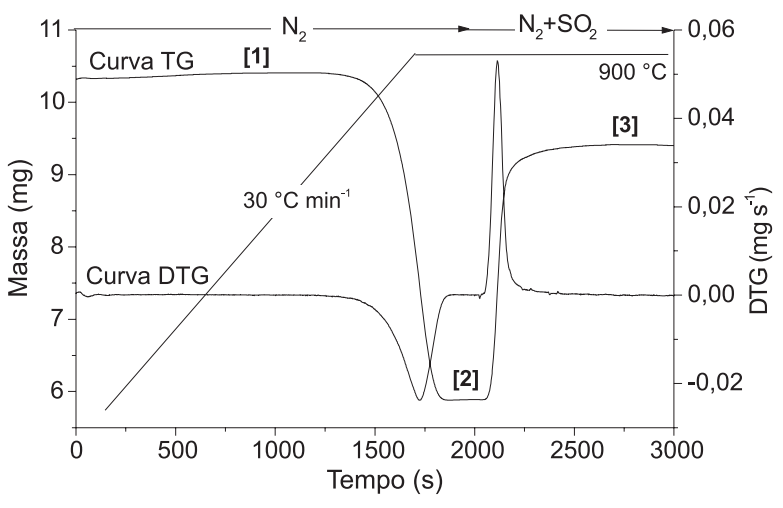

Figura 2. Curvas TG e DTG da calcinação seguida de sulfatação para um ensaio a $900{ }^{\circ} \mathrm{C}$ em atmosfera dinâmica de nitrogênio

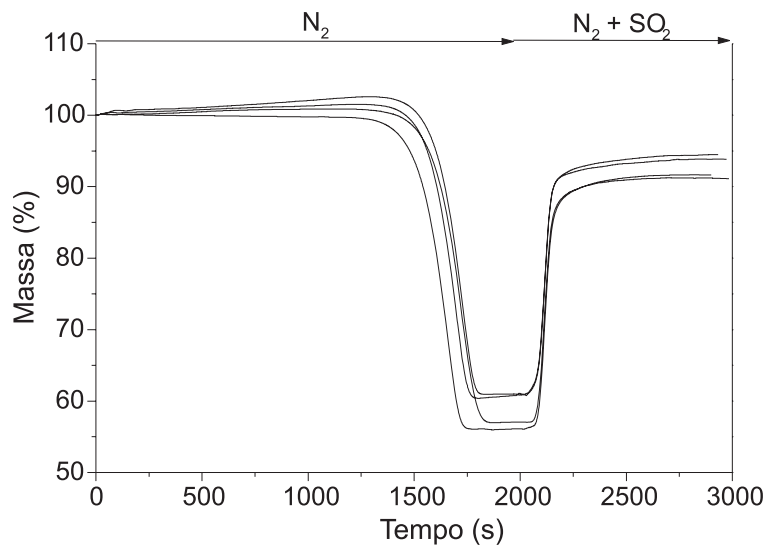

Figura 3. Conjunto de curvas TG de quatro ensaios do processo de calcinação seguida de sulfatação na temperatura de $900{ }^{\circ} \mathrm{C}$ em atmosfera de nitrogênio

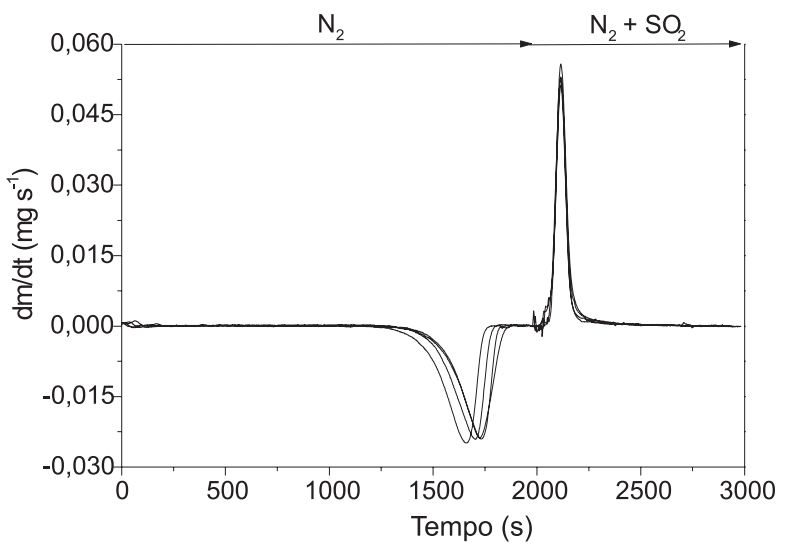

Figura 4. Conjunto de curvas DTG obtidas das curvas TG da Figura 3, para os quatro ensaios do processo de calcinação seguida de sulfatação na temperatura de $900{ }^{\circ} \mathrm{C}$ em atmosfera de nitrogênio

Em alguns ensaios observou-se nas curvas TG que antes do processo de calcinação ocorreu uma perda brusca de massa atribuída ao fenômeno da decrepitação. A Figura 5 mostra este evento para atmosfera de ar sintético, que ocorreu entre 750 e 980 s. Em atmosfera de nitrogênio foi também observado o fenômeno, que ocorreu entre 800 e 1060 s. Em atmosfera de ar sintético observou-se a decrepitação em quatro ensaios, que ocorreu no intervalo de temperatura de 380 a $465{ }^{\circ} \mathrm{C}$. Em atmosfera de nitrogênio a decrepitação foi observada também em quatro ensaios entre as temperaturas de
420 a $530{ }^{\circ} \mathrm{C}$. É importante observar que este fenômeno não interfere nos resultados da conversão na sulfatação, pois para os cálculos utiliza-se como valor da massa inicial da amostra $\left(M_{A}\right)$, a massa anterior ao início da calcinação.

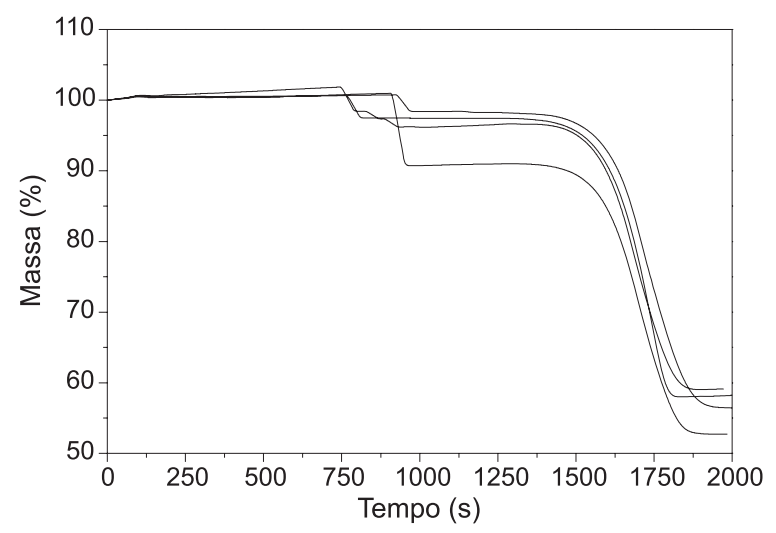

Figura 5. Fenômeno da decrepitação observado nas curvas TG de quatro ensaios obtidos em atmosfera de ar sintético entre as temperaturas de 380 a $465^{\circ} \mathrm{C}$

Nos cálculos de conversão na sulfatação convencionou-se padronizar os resultados estabelecendo-se os seguintes critérios: assumiu-se como tempo zero $\left(t_{0}\right), 100 \mathrm{~s}$ antes do pico máximo da curva DTG, e tempo final $\left(t_{\infty}\right), 500 \mathrm{~s}$ após o tempo zero. Em todos os ensaios no tempo zero a amostra encontrava-se totalmente calcinada, na temperatura de ensaio, e em atmosfera sem $\mathrm{SO}_{2}$. Observa-se que o tempo final excede aquele necessário para sulfatação total. Quando cessa a sulfatação, $d m / d t$ torna-se zero, e não há mais variação da massa da amostra no tempo, como pode ser visto nas curvas DTG da Figura 4.

Nas Figuras 6 e 7 são apresentadas as variações de massa percentual das amostras após injeção de $\mathrm{SO}_{2}$ na balança TG, nas atmosferas de ar e nitrogênio, respectivamente. Cada curva corresponde à média de quatro ensaios para cada temperatura estudada.

Observa-se que as curvas de variação de massa em todas as temperaturas estudadas apresentam comportamentos semelhantes. Em atmosfera de ar o valor final da variação de massa percentual após a sulfatação decresceu na seguinte ordem: $800 \approx 850>750>$ $900>950{ }^{\circ} \mathrm{C}$ (Figura 6). Em atmosfera de nitrogênio obteve-se a seguinte ordem: $900>950 \approx 850>800>750{ }^{\circ} \mathrm{C}$ (Figura 7). As variações de ganho de massa na sulfatação, em ambas as atmosferas, mostram uma forte dependência da temperatura.

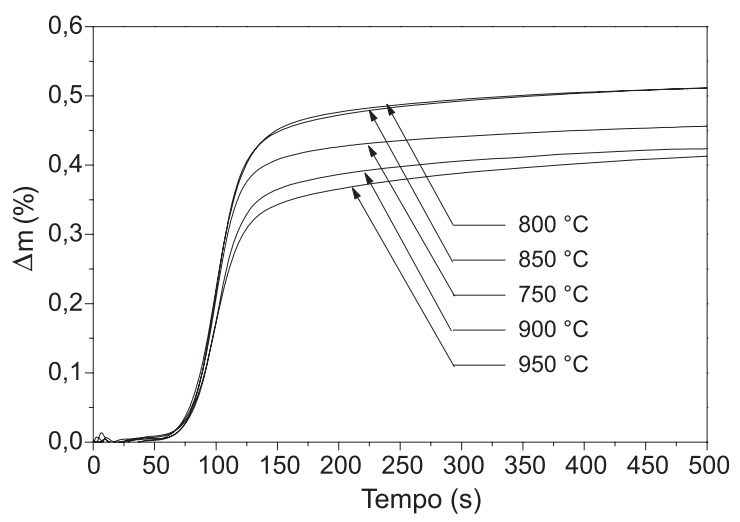

Figura 6. Valores médios da variação de massa ( $\Delta m)$ na sulfatação do calcário em função do tempo, em atmosfera de ar nas condições isotérmicas de 750, $800,850,900$ e $950^{\circ} \mathrm{C}$ 


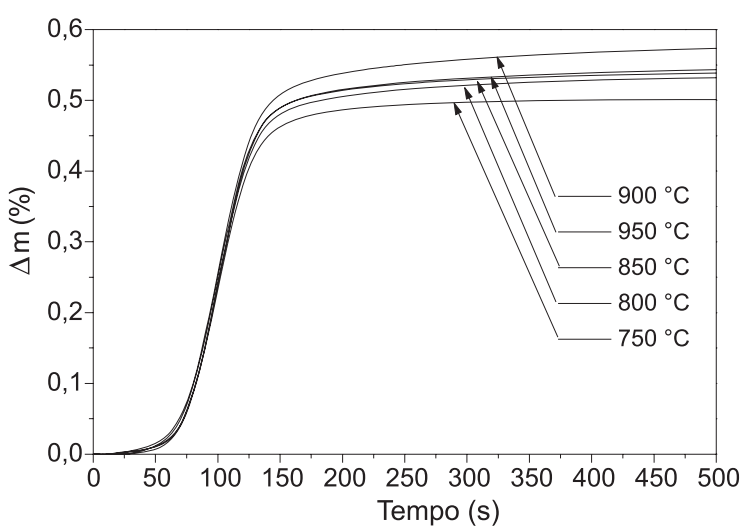

Figura 7. Valores médios da variação de massa $(\Delta m)$ na sulfatação do calcário em função do tempo, em atmosfera de nitrogênio nas condições isotérmicas de $750,800,850,900$ e $950^{\circ} \mathrm{C}$

Em atmosfera de ar, a massa final em altas temperaturas (900 e $950^{\circ} \mathrm{C}$ ) resultou inferior até mesmo ao valor encontrado para a temperatura de $750{ }^{\circ} \mathrm{C}$. Em atmosfera de nitrogênio observou-se diminuição na massa final a $950{ }^{\circ} \mathrm{C}$, quando comparada ao valor a $900{ }^{\circ} \mathrm{C}$. A redução da absorção em temperaturas mais elevadas, em ambas as atmosferas, deve-se supostamente à sinterização do sorvente. Este efeito mostrou-se muito mais intenso em atmosfera de ar.

As Figuras 6 e 7 mostram também que a absorção é mais intensa em atmosfera de nitrogênio, em todas as temperaturas. Verifica-se que em atmosfera de nitrogênio a absorção é menos sensível à temperatura que em atmosfera de ar. Atribui-se este fato possivelmente ao mecanismo da reação apresentada na rota $3^{8}$.

Com base nas curvas TG obtidas, calculou-se a conversão $(X)$ por meio das Equações 17 e 18, para as atmosferas de ar e de nitrogênio, respectivamente.

A Tabela 1 apresenta os valores de máxima conversão média percentual da absorção de enxofre pelo calcário nas duas atmosferas estudadas. Em atmosfera de ar sintético o valor médio máximo de conversão $(41,3 \%)$ ocorreu na temperatura de $850{ }^{\circ} \mathrm{C}$, enquanto que em atmosfera de nitrogênio o valor médio máximo $(58,0 \%)$ ocorreu a $900{ }^{\circ} \mathrm{C}$. Assim, levando-se em consideração estes valores médios máximos, a máxima conversão em atmosfera de nitrogênio resultou aproximadamente $29 \%$ maior que em atmosfera de ar.

Na Figura 8 são representados os valores de máxima conversão média percentual em função da temperatura, para as duas atmosferas estudadas. Nota-se que, tanto em atmosfera de ar quanto em nitrogênio, a conversão aumentou com a temperatura até um ponto de máximo, decaindo a seguir. Considerou-se o ponto de máximo do ajuste polinomial como a temperatura ótima de conversão no processo de sulfatação de calcário. A temperatura ótima de sulfatação resultou em $831{ }^{\circ} \mathrm{C}$ para atmosfera de ar, e em $894{ }^{\circ} \mathrm{C}$ para atmosfera de nitrogênio.

De modo geral, para as duas atmosferas estudadas, observou-se dispersão dos resultados em torno da média, dado pelo desvio padrão. A Tabela 2 apresenta valores dos coeficientes de variação $(C V)$ obtidos nos resultados da conversão no processo de sulfatação de calcário. Os resultados de $C V$ foram entre 3,05 e 8,80\% em atmosfera de ar sintético e, entre 2,51 e 7,36\% em atmosfera de nitrogênio. Estes valores de $C V$ podem ser considerados baixos devido a algumas particularidades, tanto do processo quanto da amostra. Trata-se de uma reação heterogênea gás-sólido em que ocorrem fatores difusionais e de transferência de transporte de massa intra-partícula. Também deve-se considerar a heterogeneidade da amostra tanto em relação à composição química quanto à distribuição granulométrica.
Tabela 1. Percentual de conversão média $\left(\mathrm{C}_{\mathrm{M}}\right)$ da absorção de enxofre pelo $\mathrm{CaO}$ em atmosferas de ar $(\mathrm{Ar})$ e nitrogênio $\left(\mathrm{N}_{2}\right)$ para diferentes temperaturas $(\mathrm{T})$

\begin{tabular}{lcc}
\hline $\mathrm{T}\left({ }^{\circ} \mathrm{C}\right)$ & $\begin{array}{c}\mathrm{C}_{\mathrm{M}}{ }^{*} \\
\mathrm{Ar}(\%)\end{array}$ & $\begin{array}{c}\mathrm{C}_{\mathrm{M}} * \\
\mathrm{~N}_{2}(\%)\end{array}$ \\
\hline 750 & 38,0 & 50,5 \\
800 & 41,1 & 54,6 \\
850 & 41,3 & 55,0 \\
900 & 36,4 & 58,0 \\
950 & 35,0 & 56,1 \\
\hline & {$\left[\frac{\mathrm{n}^{\circ} \text { moles de Ca }+ \text { Mg utilizados para absorver } \mathrm{SO}_{2}}{\mathrm{n}^{\circ} \text { moles de Ca }+ \text { Mg na amostra }} \times 100\right]$}
\end{tabular}

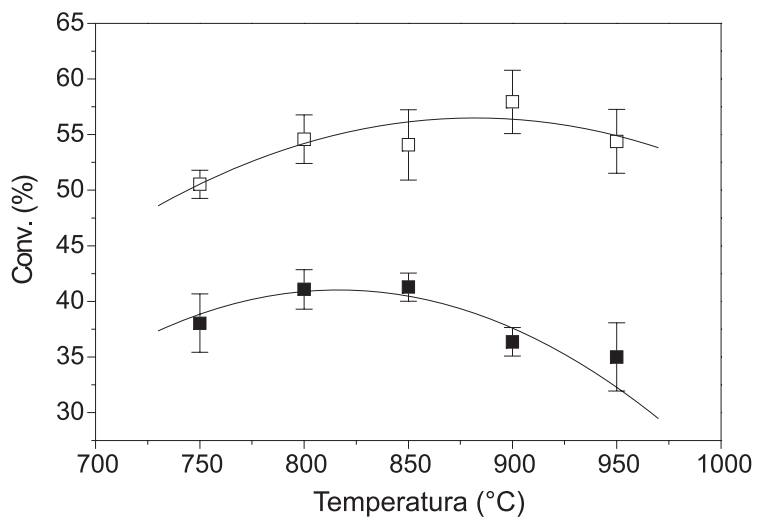

Figura 8. Conversão percentual $\left(\frac{\text { moles de } \mathrm{SO}_{2} \text { absorvido }}{\text { moles de } \mathrm{Ca}+\mathrm{Mgna} \text { amostra }} \times 100\right)$, Conv. (\%), da absorção de $\mathrm{SO}_{2}$ pelo calcário, em atmosferas dinâmicas de $\mathrm{Ar}$ (口) e de nitrogênio ( $\square$ ), em função da temperatura. As barras indicam o desvio padrão em relação à média

Tabela 2. Valores médios $(\bar{X})$ de máxima conversão, desvio padrão $(\sigma)$ e coeficiente de variação $(C V)$ obtidos em atmosferas de ar (Ar) e de nitrogênio $\left(\mathrm{N}_{2}\right)$ no processo de sulfatação para diferentes temperaturas $(\mathrm{T})$

\begin{tabular}{lcccccc}
\hline $\mathrm{T}\left({ }^{\circ} \mathrm{C}\right)$ & & $\mathrm{Ar}$ & & & $\mathrm{N}_{2}$ & \\
& $\bar{X}$ & $\sigma$ & $C V(\%)$ & $\bar{X}$ & $\sigma$ & $C V(\%)$ \\
\hline 750 & 38,0 & 2,63 & 6,92 & 50,5 & 1,27 & 2,51 \\
800 & 41,1 & 1,78 & 4,33 & 54,6 & 2,18 & 3,99 \\
850 & 41,3 & 1,26 & 3,05 & 55,0 & 4,05 & 7,36 \\
900 & 36,4 & 1,28 & 3,52 & 57,9 & 2,84 & 4,90 \\
950 & 35,0 & 3,08 & 8,80 & 56,1 & 3,82 & 6,81 \\
\hline
\end{tabular}

\section{CONCLUSÕES}

Nas duas atmosferas estudadas (ar e nitrogênio), observaramse comportamentos semelhantes entre as curvas de ganho de massa no processo de sulfatação do calcário em todas as temperaturas estudadas.

Em atmosfera de ar, o ganho de massa na sulfatação tanto a 900 quanto a $950{ }^{\circ} \mathrm{C}$ resultou inferior ao valor encontrado a $750{ }^{\circ} \mathrm{C}$. Em atmosfera de nitrogênio, o ganho de massa a $950{ }^{\circ} \mathrm{C}$ resultou inferior a $900{ }^{\circ} \mathrm{C}$. Essas quedas na absorção nas temperaturas mais elevadas devem-se, possivelmente, à sinterização das partículas do calcário.

A temperatura ótima de conversão foi de $831{ }^{\circ} \mathrm{C}$ para atmosfera de ar, e de $894{ }^{\circ} \mathrm{C}$ para atmosfera de nitrogênio. 
A absorção de $\mathrm{SO}_{2}$ mostrou-se mais intensa em atmosfera de $\mathrm{N}_{2}$ que em ar, em todas as temperaturas. Ainda em atmosfera de $\mathrm{N}_{2}$, a absorção mostrou-se menos sensível à temperatura do que em atmosfera de ar. Os máximos valores de conversão resultaram até $29 \%$ maiores em atmosfera de nitrogênio em relação à atmosfera de ar.

\section{AGRADECIMENTOS}

Este trabalho teve suporte financeiro da FAPESP (Processo 05/ 50152-5) e do CNPq.

\section{REFERENCIAS}

1. Li, Y.; Sadakata, M.; Fuel 1999, 78, 1089

2. Van Houte, G.; Delmon, B.; J. Chem. Soc., Faraday Trans. 1 1979, 75, 1593.

3. Dam-Johansen, K.; Ostergaard, K.; Chem. Eng. Sci. 1991, 46, 827.

4. Anthony, E. J.; Granatstein, D. L.; Prog. Energy Combust. Sci. 2001, 27, 215.

5. Hartman, M.; Couchlin, R. W.; AIChE J. 1976, 22, 490.

6. Simons, G. A.; Garman, A. R.; Boni, A. A.; AIChE J. 1987, 33, 211.

7. Moss, G.; Institute of fuel symposium series N.1: Fluidised Combus. D2, Institute of Fuel, London, 1975.

8. Low, M. J. D.; Goodsel, A. J.; Takezawa, N.; Environ. Sci. Technol. 1971, 5,1191 .

9. Borgwardt, R. H.; Bruce, K. R.; AIChE J. 1986, 32, 239.

10. Adánez, J.; Garciá-Labiano, F.; Gayán, P.; Fuel Process. Technol. 1993, 36, 73.
11. Yrjas, P.; Iisa, K.; Hupa, M.; Fuel 1995, 74, 395.

12. Silva, F. F.; Dissertação de Mestrado, Universidade de São Paulo, Brasil, 2003.

13. Kato, K.; Sakamoto, H.; Sakurai, H.; Takarada, T.; Nakagawa, N.; J. Chem. Eng. Jpn. 1994, 27, 276.

14. Mcintosh, R. M.; Sharp, J. H.; Wilburn, F. W.; Thermochim. Acta 1990 , 165, 281.

15. McCauley, R. A.; Johnson, L. A.; Thermochim. Acta 1991, 185, 271.

16. Crnkovic, P. C. G. M.; Tese de Doutorado, Universidade de São Paulo, Brasil, 2003.

17. Crnkovic, P. M.; Polito, W. L.; Silva Filho, C. G.; Milioli, F. E.; Quim. Nova 2004, 27, 58.

18. Pettijohn, F. J.; Sedimentary Rocks. Harper: New York, 1957.

19. Siegel, S.; Fuchs, L. H.; Hubble, B. R.; Nielsen, E. L.; Environ. Sci. Technol. 1978, 12, 1411

20. Zevenhoven, R.; Yrjas, P.; Huppa, M.; Fuel 1998, 77, 285.

21. Hartman, M.; Svoboda, K.; Trka, O.; Vessely, V.; Chem. Eng. Sci. 1988, 43, 2045.

22. Hartman, M.; Svoboda, K.; Ind. Eng. Chem. Process Des. Dev. 1985, 24, 613.

23. Camargo, F. L.; Dissertação de Mestrado, Universidade de São Paulo, Brasil, 2001.

24. Liping, C.; Weiren, B.; Kechang, X.; Energy Sources 2001, 23, 287.

25. Dennis, S.; Hayhurst, A. N.; Chem. Eng. Sci. 1990, 45, 1175.

26. Wieczorek-Ciurowa, K.; J. Therm. Anal. 1992, 38, 2101.

27. Leite, F.; Validação em Análise Química, Ed. Átomo: Campinas, 1996.

28. Gomes, F. P. C.; Curso de Estatística Experimental, 5ª . ed., Livraria Nobel S.A: São Paulo, 1973.

29. Ávila, I.; Dissertação de Mestrado, Universidade de São Paulo, Brasil, 2005. 